\title{
Portograma aéreo y neumatosis intestinal como hallazgos incidentales en postoperatorio de cirugía abdominal
}

\author{
Laura Morales $C^{1}$, Jorge Carrillo $B^{2}$, Juan Sebastián Ojeda $G^{3}$, Germán Torres A $^{4}$.
}

1. Médico General, Universidad del Rosario, Bogotá, Colombia.

2. Medico Radiólogo, Hospital Universitario Mayor - Méderi, Bogotá, Colombia.

3. Médico General, Universidad del Rosario, Bogotá, Colombia.

4. Médico cirujano general, Hospital Universitario Mayor - Méderi, Bogotá, Colombia.

\section{Hepatic portal venous gas and pneumatosis intestinalis as incidental findings in post-surgical abdominal surgery}

Abstract. Hepatic portal venous gas (HPVG) is defined as the presence of air in the portal venous system. Pneumatosis intestinalis (PI) is defined as the presence of air within the bowel wall, regardless of its cause or location. Its main etiology is the intestinal ischemia and are generally considered predictors of intestinal perforation and wrong prognosis. A small group of patients with HPVG and PI may have a different clinical course, without complications and clinical manifestations.

We report the case of a patient with immediate surgical history of total gastrectomy and Roux-en-Y reconstruction, which showed in computed tomography (CT) of the abdomen HPVG and PI, without associated clinically significant changes.

Keywords: Hepatic portal venous gas, Pneumatosis Cystoides Intestinalis, intestinal ischemia, gastrectomy.

Resumen. Portograma aéreo o portograma de aire (PA), se define como la presencia de aire en el sistema venoso portomesentérico. Neumatosis intestinal (NI) se define como la presencia de aire en la pared intestinal, independiente de su causa o localización. La principal etiología de estas alteraciones es la isquemia intestinal aguda y en general, se consideran predictores de perforación intestinal y de mal pronóstico. Un pequeño grupo de pacientes con PA y/o NI pueden evolucionar sin complicaciones e incluso cursan sin manifestaciones clínicas.

Presentamos el caso de una paciente con antecedente quirúrgico inmediato de gastrectomía total y reconstrucción en Y de Roux, que evidenció en tomografía computarizada (TC) de abdomen de control PA y NI, sin alteraciones clínicas significativas asociadas.

Palabras clave: Portograma aéreo, neumatosis intestinal, isquemia intestinal, gastrectomia.

Morales L., et al. Portograma aéreo y neumatosis intestinal como hallazgos incidentales en postoperatorio de cirugía abdominal. Rev Chil Radiol 2018; 24(1): 40-43.

Correspondencia: Laura Morales C. / lauracristina25@hotmail.com

Trabajo enviado el 23 de febrero de 2018. Aceptado para publicación el 18 de mayo de 2018.

\section{Presentación del caso}

Mujer de 62 años con antecedente quirúrgico inmediato de gastrectomía total y reconstrucción en $\mathrm{Y}$ de Roux por diagnóstico de adenocarcinoma gástrico tipo intestinal (Bormann III).

Por dolor abdominal en el día 5 del postoperatorio, se practicó TC contrastada de abdomen, que evidenció PA y NI, sin otros hallazgos de importancia (Figuras 1, 2 y 3). El manejo realizado incluyó: reanimación hídrica, analgesia y retiro de la sonda de nutrición enteral.

El dolor abdominal cedió con el manejo descrito. La evolución clínica fue satisfactoria, por lo que no se realizó control imagenológico, fue dada de alta 3 días después del cuadro de dolor. El control ambulatorio a los 5 meses confirmó una adecuada evolución clínica.

\section{Discusión}

El portograma aéreo (PA) es un hallazgo radiológico poco frecuente, se utiliza para describir la presencia de aire en el sistema venoso portomesentérico. El término de neumatosis intestinal $(\mathrm{NI})$ corresponde a la presencia de aire en la pared intestinal, de localización mucosa, submucosa o serosa que puede presentarse en cualquier segmento del tracto digestivo $0^{1,2,3,4,5}$. 


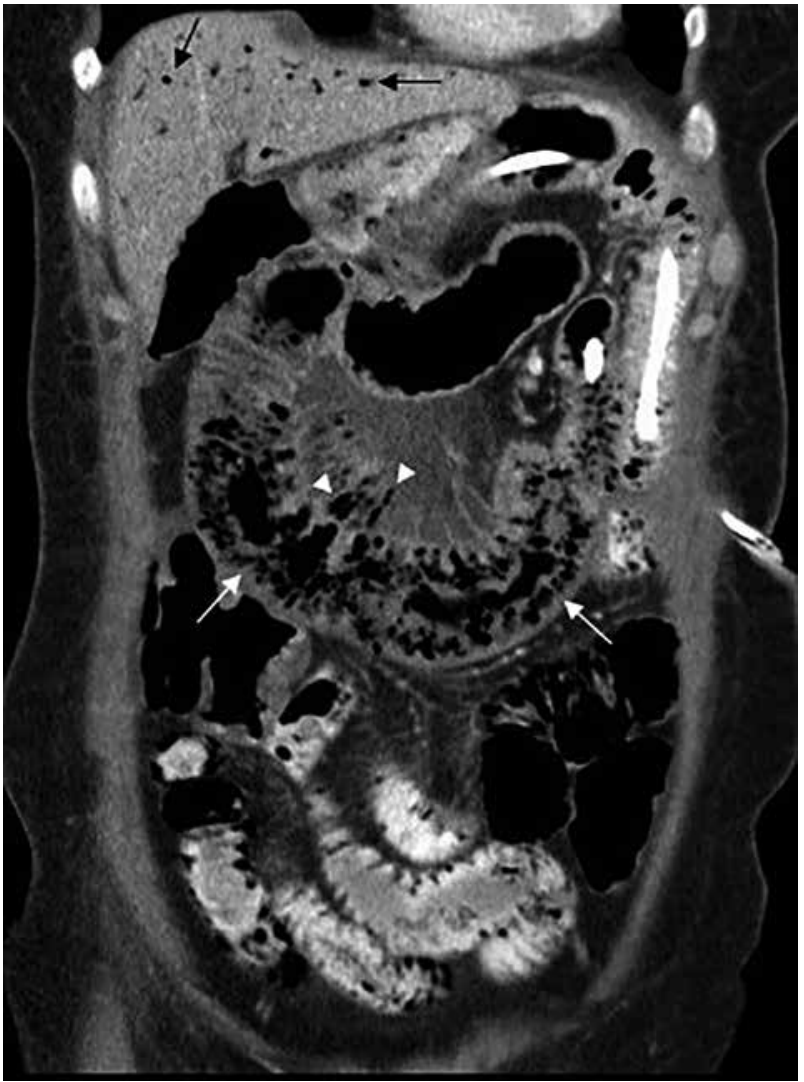

Figura 1. TC de abdomen. Reconstrucción coronal. Portograma de aire (flechas negras), neumatosis intestinal (flechas blancas) y aire en la luz de vasos mesentéricos (punta de flecha).

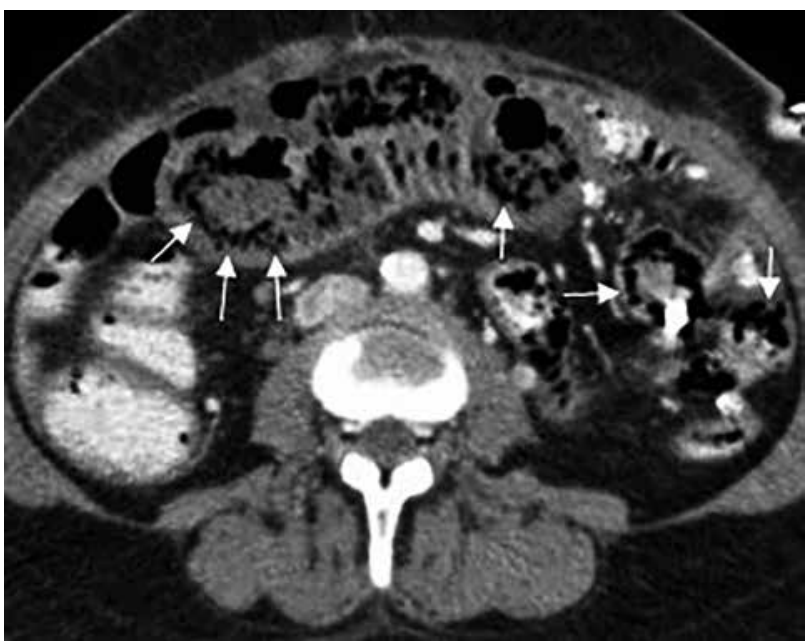

Figura 2. TC de abdomen. Aire en la pared de asa yeyunal en mesogastrio correspondiente a neumatosis intestinal (flechas blancas).

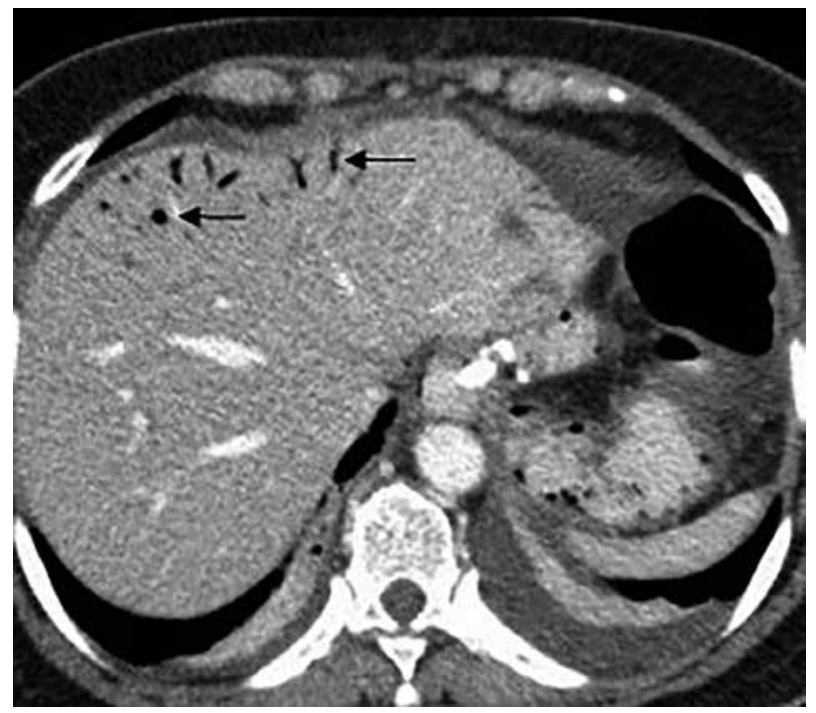

Figura 3. TC de abdomen. Imágenes lineales hipodensas, subcapsulares hepáticas, correspondientes a portograma de aire (flechas negras).

En general, estas alteraciones se presentan entre la quinta y octava década de la vida. Su etiología se clasifica en primaria y secundaria ${ }^{2}$. La primaria 0 idiopática (15\%), se presenta sin síntomas asociados y se considera un hallazgo incidental en los estudios de imágenes ${ }^{2,6}$. La secundaria (85\%), se relaciona con condiciones de naturaleza diversa que se pueden agrupar en 3 grandes grupos. Causas isquémicas: isquemia mesentérica o infarto intestinal. Causas no isquémicas: obstrucción intestinal, íleo paralítico, ulcera gástrica perforada, carcinomas (colon), trauma abdominal, descompresión hiperbárica, procesos inflamatorios intraabdominales (enfermedad de Crohn, colitis ulcerativa, apendicitis, diverticulitis, pancreatitis, ingestión de cáusticos), entidades infecciosas intraabdominales (colangitis, pileflebitis, abscesos, tuberculosis e infección por Clostridium difficile), enfermedades autoinmunes (esclerodermia, lupus eritematoso sistémico, dermatomiositis, sarcoidosis), procedimientos quirúrgicos abdominales y medicamentos (esteroides, quimioterapia, inhibidores de la a-glucosidasa). Causas iatrogénicas: procedimientos endoscópicos, colangiopancreatografía endoscópica retrograda, estudios baritados, sondas de nutrición enteral ${ }^{2,4,5,7,8}$.

La isquemia intestinal aguda, es la causa más importante de PA y/o NI, con peor pronóstico y mortalidades descritas hasta del $80-90 \% \%^{3,5,7,8,9}$. La probabilidad de isquemia intestinal aumenta significativamente cuando la $\mathrm{NI}$ se acompaña de aire en el sistema venoso portomesentérico ${ }^{5}$. La presencia de PA se consideraba previamente como signo de necrosis intestinal que justificaba la necesidad de 
cirugía urgente ${ }^{8}$. La mortalidad reportada por PA ha disminuido, pasando de un $75 \%$ en los primeros estudios realizados en $1978^{10}$ a $27,3 \%$ en un estudio reciente realizado en Japón, el cual involucro 1950 pacientes con diagnóstico de $\mathrm{PA}^{11}$.

La fisiopatología de estos hallazgos se considera multifactorial ${ }^{5,12}$. La disrupción de la integridad de la mucosa es una característica común a todas las teorías postuladas 5 . Se describen tres teorías: a) Bacteriana. Propone que el gas de la pared intestinal se relaciona con la presencia de microorganismos anaerobios productores de gas. b) Mecánica. Propone que el gas penetra en la pared intestinal por trauma directo o por aumento exagerado de la presión intraluminal y c) Lesión mucosa. Propone que el principal mecanismo es la lesión de la mucosa intestinal, que permite la entrada de bacterias o aire a la pared intestinal y posteriormente entra al sistema venoso portal ${ }^{2,4,5,7 .} \mathrm{La}$ teoría de la lesión mucosa parece la mejor explicación para la NI asociada a inflamación, isquemia, uso de esteroides y antecedente de quimioterapia ${ }^{2,5}$.

En el contexto de un paciente en postoperatorio de cirugía abdominal, la presencia de PA y NI sugieren isquemia intestinal. La necrosis intestinal en estos pacientes se relaciona con deshidratación intravascular secundaria al aumento de la permeabilidad vascular (mediada por citoquinas inflamatorias y estrés inherente al procedimiento quirúrgico) y aumento de la proliferación bacteriana y de la producción de gas intestinal, que condicionan incremento de la presión intraluminal ${ }^{1}$. Otros factores de riesgo incluyen: lesión intestinal durante la cirugía y complicaciones relacionadas con las anastomosis quirúrgicas ${ }^{1}$.

Las manifestaciones clínicas varían de acuerdo con la etiología de PA y NI. Una condición crítica como isquemia mesentérica, se presenta con signos de abdomen agudo, choque e hiperlactatemia ${ }^{9,13,14}$. Por otra parte, las etiologías benignas cursan con signos y síntomas menos específicos como diarrea o estreñimiento y dolor abdominal entre otros. Como mencionamos previamente un grupo de pacientes con PA y NI, cursan sin sintomatología9.

En la valoración de pacientes con sospecha de isquemia intestinal la TC es el método de elección de diagnóstico por imagen, con mayor sensibilidad y especificidad en la detección de alteraciones vasculares y de la pared intestinal ${ }^{15,16}$. La TC permite detectar PA

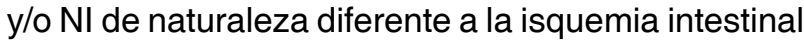
con ventaja sobre las demás modalidades de imágenes $^{16}$. La radiografía de abdomen y la ultrasonografía abdominal tienen un rendimiento inferior a la TC para demostrar PA y/o NI y una utilidad limitada para detectar signos primarios o secundarios de isquemia intestinal ${ }^{16,17}$.

El portograma aéreo en la TC de abdomen se caracteriza por la presencia de imágenes tubulares de baja atenuación que alcanzan la periferia del parénquima hepático. En la radiografía de abdomen el PA se visualiza como imágenes lineales radiolúcidas que pueden alcanzar la periferia hepática ${ }^{3,17}$. En ultrasonografía el aire portal puede presentar tres patrones imagenológicos: 1) Patrón de puntos (asociado a gas en escasa cantidad) y representado por focos ecogénicos, móviles, de distribución ramificada en el lumen de la vena porta ; 2) Patrón de rayas (asociado a mayor cantidad de gas) y representado por imágenes lineales hiperecoicas de distribución radiada en el parénquima hepático que se extienden a la periferia del hígado y 3 ) Patrón de pulpa de fruta, que semeja el corte transversal de un cítrico, asociado a mayor cantidad de gas y visualizado como imágenes ecogénicas parenquimatosas con tendencia a la confluencia, que se extienden a la región subcapsular ${ }^{15,18}$. En el diagnóstico diferencial de PA se debe considerar la presencia de aire en la vía biliar (neumobilia) ${ }^{3}$. La neumobilia en TC se caracteriza por la presencia de imágenes tubulares ramificadas, adyacentes a las ramas portales, de distribución central, que confluyen en el conducto hepático común ${ }^{3,17}$.

El único hallazgo radiológico que ha demostrado tener una adecuada correlación con el origen isquémico del PA y la NI, es la disminución del realce de un segmento intestinal ${ }^{5}$. La presencia de estos hallazgos imagenológicos, no son indicadores de resección quirúrgica per $\mathrm{se}^{8}$. La extensión, severidad, distribución y características morfológicas de la NI (quística o lineal), no se consideran factores predictores de mal pronóstico $2,5,7,9$. Por ende, la importancia de los signos radiológicos y su significado, requieren un conocimiento preciso del contexto clínico del paciente y no deben ser interpretados de manera aislada.

\section{Conclusión}

La paciente presentó una adecuada evolución clínica, sin complicaciones relevantes a pesar de la presencia de PA y $\mathrm{NI}$ en la TC de abdomen. Los hallazgos radiológicos no siempre sugieren la presencia de etiología critica como la isquemia mesentérica. En el caso presentado, la presencia de PA y NI pudieron estar relacionados con el procedimiento quirúrgico abdominal (gastrectomía total y reconstrucción en $Y$ de Roux) y la presencia de sonda nutricional enteral en el postoperatorio. La evolución clínica fue satisfactoria con el manejo medico recibido por lo cual no se realizó control imagenológico. El control ambulatorio confirmo la adecuada evolución.

\section{Referencias}

1. Higashizono K, Yano $\mathrm{H}$, Miyake $\mathrm{O}$, Yamasawa $\mathrm{K}$, Hashimoto M. Postoperative pneumatosis intestinalis (PI) and portal venous gas (PVG) may indicate bowel necrosis: A 52-case study. BMC surgery. 2016; 16(1): 42. 
2. Feczko P, Mezwa DG, Farah M, White B. Clinical significance of pneumatosis of the bowel wall. Radiographics. 1992; 12(6): 1069-1078.

3. Castren EE, Hakeem AR, Mahmood NS, Aryal K. Case of pneumatosis intestinalis and hepatic portal venous gas following a laparoscopic right hemicolectomy. BMJ case reports. 2016; 2016: bcr2016214431.

4. Li Z, Su Y, Wang X, Yan H, Sun M, Shu Z. Hepatic portal venous gas associated with colon cancer: A case report and literature review. Medicine. 2017; 96(50): e9352.

5. Treyaud M-O, Duran R, Zins M, Knebel J-F, Meuli RA, Schmidt S. Clinical significance of pneumatosis intestinaliscorrelation of MDCT-findings with treatment and outcome. European radiology. 2017; 27(1): 70-79.

6. Akimori T, Kanagawa T, Fujisawa K, Kamioka N, Miyazaki J, Usui T, et al. Pneumatosis intestinalis: report of three cases with different clinical features. Annals of Cancer Research and Therapy. 2015; 23(1): 5-8.

7. Pizarro A, Schnellenkamp K, Melgarejo S. Caso clínico radiológico para diagnóstico. Revista chilena de radiología. 2009; 15(2): 95-98.

8. Iwai N, Handa O, Naito Y, Dohi O, Okayama T, Yoshida N, et al. Stenotic Ischemic Enteritis with Concomitant Hepatic Portal Venous Gas and Pneumatosis Cystoides Intestinalis: A Case Report. Internal Medicine. 2018: 0367-17.

9. Zorgdrager M, Pol R. Pneumatosis intestinalis associated with enteral tube feeding. BMJ case reports. 2013; 2013: bcr2013009378.

10. Liebman PR, Patten MT, Manny J, Benfield JR, Hechtman HB. Hepatic--portal venous gas in adults: etiology, pathophysiology and clinical significance. Annals of surgery. 1978; 187(3): 281.
11. Koizumi C, Michihata N, Matsui H, Fushimi K, Yasunaga $\mathrm{H}$. In-hospital mortality for hepatic portal venous gas: analysis of 1590 patients using a Japanese National Inpatient Database. World journal of surgery. 2018; 42(3): 816-822.

12. Blair HA, Baker R, Albazaz R. Pneumatosis intestinalis an increasingly common radiological finding, benign or life-threatening? A case series. BMJ case reports. 2015; 2015: bcr2014207234.

13. Ho LM, Paulson EK, Thompson WM. Pneumatosis intestinalis in the adult: benign to life-threatening causes. American Journal of Roentgenology. 2007; 188(6): 1604-1613.

14. Duron VP, Rutigliano S, Machan JT, Dupuy DE, Mazzaglia PJ. Computed tomographic diagnosis of pneumatosis intestinalis: clinical measures predictive of the need for surgical intervention. Archives of Surgery. 2011; 146(5): 506-510.

15. Piton G, Capellier G, Delabrousse E. Echography of the portal vein in a patient with shock. Critical care medicine. 2016;44(6): e443-e5.

16. Oliva IB, Davarpanah AH, Rybicki FJ, Desjardins B, Flamm SD, Francois CJ, et al. ACR Appropriateness Criteria ${ }^{\circledR}$ imaging of mesenteric ischemia. Abdominal imaging. 2013; 38(4): 714-719.

17. Sebastià $C$, Quiroga $S$, Espin $E$, Boyé $R$, Alvarez-Castells A, Armengol M. Portomesenteric vein gas: pathologic mechanisms, CT findings, and prognosis. Radiographics. 2000; 20(5): 1213-1224.

18. Pan H-B, Huang J-S, Yang T-L, Liang H-L. Hepatic portal venous gas in ultrasonogram-benign or noxious. Ultrasound in Medicine and Biology. 2007; 33(8): 1179-1183. 